\title{
Quantitative Visualization Analysis in the Field of Sports Dance Based on CiteSpace
}

\author{
Dacai Huang \\ Shenyang Sport University, Shenyang 110100, Liaoning, China \\ hdcai1996@yeah.net
}

\begin{abstract}
Taking 266 CSSCI records of sports dance from CNKI database as the research object, the relevant data were analyzed by CiteSpace software. By means of knowledge map, this paper combs the number of papers, high-yield institutions, high-yield authors, cooperation and research hotspots. This study draws the following conclusions, which are divided into three time periods according to the amount of documents issued; The cooperation between the authors is scattered, and the representatives are mainly concentrated in Beijing Sports University; The distribution of research institutions is scattered and the cluster scale is small; The keywords are clustered into nine cluster labels. Through sorting and summarizing, the nine clusters are summarized into six aspects: the development of sports dance, the development of sports dance in China's mass sports, sports dance teaching, the cultural integration of national standard dance and Chinese classical dance, the aesthetic value of sports dance, and the impact of sports dance on College Students'physical and mental health.
\end{abstract}

Keywords: Sports dance, CiteSpace, Research review, Visual analysis.

\section{Introduction}

Sports dance, also known as international standard dance, is a normative and procedural sports project completed by men and women in cooperation, correctly displaying and using body technology and skills within the defined music and rhythm range, and combined with artistic expression [1]. If you want to understand the field of sports dance, only by fully grasping the research trend of sports dance in China, can you better promote the development of sports dance projects. Therefore, this paper uses the method of scientific knowledge map to analyze the relevant literature of sports dance research in China, in order to fully understand the development process, characteristics and hot spots of sports dance research in China.

\section{Data Sources and Methods}

\subsection{Data Sources}

In order to understand the research hotspots and research progress of domestic sports dance, this paper takes CNKI as the data source. In order to ensure that the relevant literature is representative, this study selects CSSCI journal as the source. The retrieval time span is set to 1991-2021, and the retrieval will be carried out on March 31, 2021. 269 academic journal papers were obtained. Through the screening of relevant literature, 266 academic journal papers were obtained as samples. It was found that the literature on Sports Dance included by CSSCI began in 1998 and the deadline of the latest literature was 2020.

\subsection{Methods}

In this paper, CiteSpace software version 5.7.r5 is used to analyze the literature. The software can analyze the author, organization and keywords, and then clearly see the cooperation, theme hotspots and other information of relevant research for a certain period of time.

\section{Results and Analysis}

\subsection{Temporal and Spatial Knowledge Map of Sports Dance Research}

\subsubsection{Time distribution of sports dance research}

The author arranges the annual distribution of the main literature on sports dance research through CNKI database. The specific data are shown in Figure 1. As shown in Figure 1, it is clear that sports dance related research first appeared in CSSCI data source in 1998; From 1998 to 2009, the overall number of documents was wavy and slowly upward; 2010 was the year with the highest number of documents in more than 20 years. Through consulting relevant materials, it can be found that at the 2010 Guangzhou Asian Games, sports dance became an official event of the Asian Games for the first time. In this game, the Chinese team created a grand slam record and won all 10 gold medals. Therefore, sports dance has become a hot topic in sports in 2010, This development trend has brought about the rapid development of relevant research. By looking at the relevant literature of that year, it is found that there are many articles on sports dance teaching and promotion; However, after that, the number of documents issued each year decreased in a wavy manner, but the decline was small. Through the visual analysis of HowNet, it is predicted that the number of documents issued in 2021 should be no different from that in 2020 , about 8 .

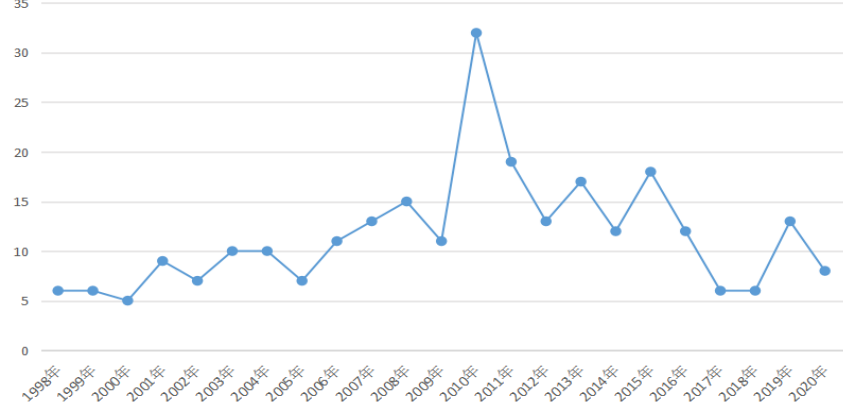

Figure 1: Chronological distribution of sports dance research literature 


\subsubsection{Spatial distribution map of sports dance research}

(1) Analysis of the main authors of sports dance research

In CiteSpace 5.7, the author is selected as the network node, and the relevant authors of sports dance research are visually analyzed to obtain the map of scientific knowledge (Figure 2).

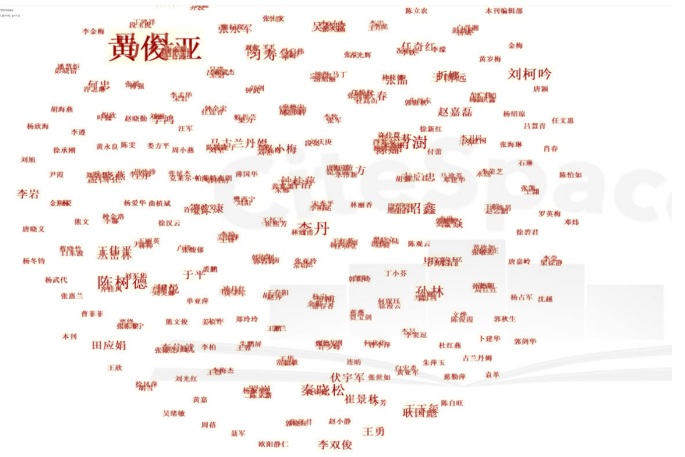

Figure 2: Cluster distribution of sports dance research authors

In the process of author clustering, it can be seen from the clustering results that $\mathrm{n}$ (total number of authors) is 371 , e (number of author cooperative relationships) is 259 , the cooperation density is 0.0038 , the clustering module value $(\mathrm{q}=0.9866)$ and the clustering average contour value $(\mathrm{s}=1)$, indicating that the clustering structure is significant and persuasive. It can be clearly seen from Figure 2 that the cooperation among authors is relatively scattered, the cooperation of small-scale authors is still clear, mainly the cooperation of 2-3 people. Table 1 lists the list of 12 scholars who have published more than 3 articles, among which Huang Junya, Ma Dan, Zhang Qingshu, Chen Shude and Li Dan rank first.

Table 1: Core authors of sports dance research and the number of papers published

\begin{tabular}{cc}
\hline Scholar & Number \\
\hline Huang Junya, Ma Dan, Zhang Qingshu, & \\
Chen Shude, Li Dan & 4 \\
Li Min, Meng Zhaoxin, Qin Xiaosong, Xi \\
Shouhua, Chen Qin, Sun Lin, Liu keyin
\end{tabular}

(2) Institutional Analysis of sports dance research

In CiteSpace 5.7, institutions are selected as network nodes to visually analyze the relevant authors of sports dance research and obtain the map of scientific knowledge (Figure 3).

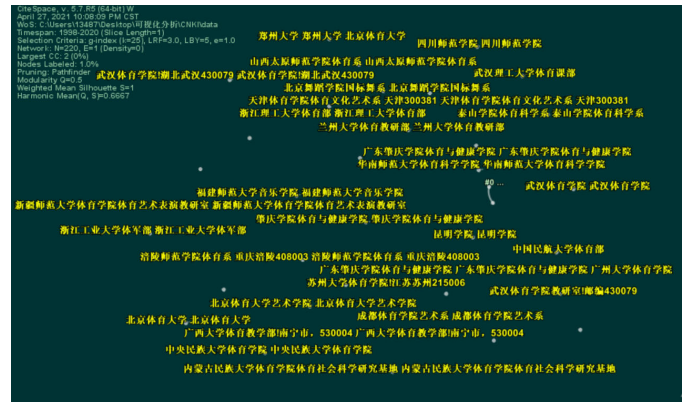

Figure 3: Cluster distribution of sports dance research institutions

In the map, we can see that $\mathrm{n}$ (total number of institutions) is 220, e (number of institutional partnerships) is 1 , the institutional cooperation density is $0, Q$ value is 0.5 , and $s$ value is 1 , indicating that the network co-occurrence structure of sports dance related institutions is significant. Although the total number of institutions is 220, there is little cooperation between institutions. Sports dance institutions are scattered and the cluster scale is small. As a result, the sharing and mobility of sports dance related knowledge and research results are not strong.

Table 2 lists 22 institutions with more than 3 articles. It can be seen from table 2 that the research on sports dance is mainly concentrated in sports colleges and universities.

Table 2: Main institutions and documents of sports dance research

\begin{tabular}{cc}
\hline Mechanism & Number \\
\hline Beijing Sport University & 21 \\
Wuhan Sport University & 18 \\
Chengdu Sport University & 10 \\
Shanghai Sport University, Tianjin Sport University & 8 \\
Guangzhou Sport University & 7 \\
South China Normal University & 6 \\
Xinjiang Normal university & 5 \\
Qufu Normal University, Zhaoqing University, Henan & 4 \\
University, Lanzhou University & \\
Southwest Jiaotong University, Henan Normal University, & \\
Wuhan University of technology, Guangzhou University of \\
traditional Chinese medicine, Fujian Normal University, Suzhou \\
University, Shenyang Conservatory of Music, Central University \\
for Nationalities, Beijing Dance Academy, Yunnan Normal \\
University \\
\hline
\end{tabular}

\subsection{Keyword Cluster Analysis of Sports Dance Research}

In CiteSpace, select keywords as network nodes, visually analyze the keywords of sports dance research, cluster and name them with keywords, and draw a map of scientific knowledge (Figure 4), so as to understand the research hotspots in the field of sports dance.

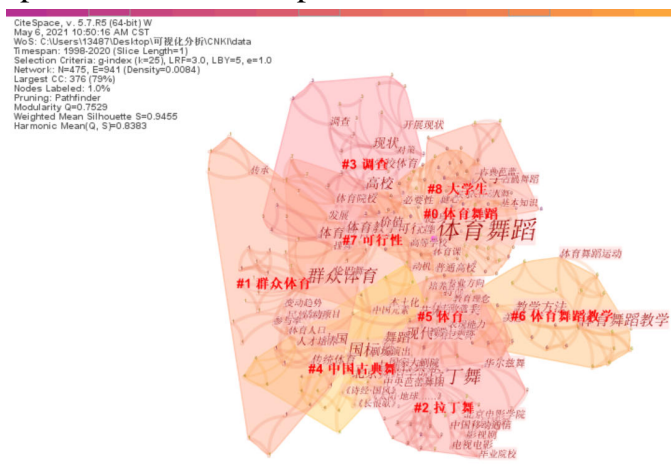

Figure 4: Clustering diagram of sports dance research keywords

In the process of mapping, it is found that $n$ (total number of keywords) is 475 and $E$ (number of relationships between keywords) is 941 , in which the keyword density is 0.0084 , the clustering module value $(\mathrm{q}=0.7529)$ and the clustering average contour value $(s=0.9455)$, which means that the network density is high and the clustering structure is significant and persuasive. Therefore, it can be seen that the relationship between keywords is relatively close. Through keyword clustering, this study finally obtains 9 clusters (see Figure 4). The 9 cluster labels are "Sports Dance", "mass sports", "Latin dance", "investigation", "Chinese classical dance", "Sports", "sports dance teaching", "feasibility" and "college students". 
Based on the keyword clustering map, the keyword co-occurrence network clustering table is obtained through software analysis (see Table 3). It includes the cluster number, cluster size, cluster name and the main identification words of related clusters.

Table 3: Sports Dance keyword co-occurrence network clustering table

\begin{tabular}{|c|c|c|c|}
\hline Cluster number & Cluster size & Cluster name & Identifier \\
\hline 0 & 74 & Sports Dance & $\begin{array}{l}\text { Sports Dance, restrictive factors, colleges and universities, teaching means, learning } \\
\text { interest, countermeasure research, communication practice, development status, } \\
\text { sunshine sports }\end{array}$ \\
\hline 1 & 40 & Mass sports & $\begin{array}{l}\text { Mass sports, China, corporate culture, aerobics and dance, change trend, traditional } \\
\text { sports, market mechanism, comparative sports, national dance, sports industry }\end{array}$ \\
\hline 2 & 36 & Latin dance & $\begin{array}{l}\text { Latin dance, training, basic movements, attention to stability, mixed teaching mode, } \\
\text { preschool, school art education, social dance, characteristic theory, control group, } \\
\text { experimental group, students' comprehensive quality and competitive ability }\end{array}$ \\
\hline 3 & 36 & Investigation & $\begin{array}{l}\text { investigation, universities, physical education, current situation, teaching effect, teaching } \\
\text { reform, sports dancers, movement elements, rhythm teaching, development, School } \\
\text { Physical Education }\end{array}$ \\
\hline 4 & 30 & Chinese classical dance & $\begin{array}{l}\text { Chinese classical dance, Beijing Dance Academy, national standard dance, professional } \\
\text { construction, Wang Qiyao, "book of songs - national style", Central Ballet, Wang } \\
\text { Wenwei, theater performance, textbook construction, discipline construction, musical, } \\
\text { collaborative governance, cultural integration and localization }\end{array}$ \\
\hline 5 & 27 & Sports & $\begin{array}{l}\text { Sports, art, aesthetics, teaching and training, characteristics, music beauty, expressive } \\
\text { ability, body culture, body beauty, professional direction, professional players, } \\
\text { educational concept, aesthetics, competitive value orientation, sports beauty and training } \\
\text { objectives }\end{array}$ \\
\hline 6 & 26 & Sports Dance Teaching & $\begin{array}{l}\text { Sports Dance teaching, teaching methods, teaching experience analysis, innovation and } \\
\text { application, technical skill teaching, sports dance education, teaching content, sports } \\
\text { dance movement, information transmission }\end{array}$ \\
\hline 7 & 24 & Feasibility & $\begin{array}{l}\text { Feasibility, colleges and universities, music quality training, heart rate, value, necessity } \\
\text { and importance analysis, "music knowledge and sports music", colleges and universities, } \\
\text { sports music, physical education, higher vocational colleges, sports fitness and dance } \\
\text { courses, sports performance and music knowledge }\end{array}$ \\
\hline 8 & 20 & College student & $\begin{array}{l}\text { College students, entertainment, emotion, mental sub-health, fitness, dance therapy, } \\
\text { interpersonal communication, coping style, acceptance, intermediary effect, healthy } \\
\text { heart and beautiful body }\end{array}$ \\
\hline
\end{tabular}

Through the keyword co-occurrence network cluster analysis of sports dance research in Figure 4 and Table 3, the existing research contents of sports dance at this stage are roughly summarized into the following six aspects:

1) The development of sports dance. The key words in this aspect are clustered as label 0 , in which the identification words mainly include "sports dance, colleges and universities, countermeasure research, teaching means, learning interest, communication practice, development status, restrictive factors, sunshine sports", etc. The research on sports dance is mainly concentrated before 2010 . In the development process of sports dance, relevant scholars first analyzed the restrictive factors existing in the development of sports dance, then carried out countermeasure research, and finally implemented communication practice. Through this process, sports dance has developed rapidly. Through the inspection of relevant literature, the relevant research of sports dance is mainly aimed at colleges and universities, this is because sports dance was first developed in Colleges and universities. In 1987, China's State Education Commission set up sports professional dance courses and sports dance elective courses in Physical Education in Colleges and universities.

2) The development of sports dance in mass sports in China. The keyword cluster is label 1, in which the identification words mainly include "mass sports, China, corporate culture, aerobics and dance, change trend, traditional sports, market mechanism, comparative sports, national dance, sports industry", etc. In order to promote the spread of sports dance in our society and make the public accept this sports project spread abroad to China, some domestic scholars have conducted relevant research on the development of sports dance in mass sports in our country. For example, Sun Lin and others discussed the development of sports dance events in China [2] and compared the development of sports dance brand events at home and abroad [3]. Bu Jianhua sought the market path of sports dance from the perspective of supply and demand theory [4]. Through these relevant studies, it can be found that such articles are mainly concentrated from 2010 to 2015. Sports dance should be divided into two categories: mass and competitive. Only in this way can it be popularized and improved in our country. Since the introduction of line dance in 2008, China has made great efforts to promote it in national fitness activities. Line dance includes all kinds of dances and exercises. Classified by style, modern dance and Latin dance belong to two parts of line dance. Through line dance, it has greatly promoted the spread of sports dance in mass sports. In the development of sports dance, it is necessary to build events belonging to China's sports dance brand, because the brand is an intangible asset and can play an important role in the organization, consumers and other relevant social groups. Sports dance has formed an industrial chain with sports events as the core, and sports events are the main driving force on the road of sports dance marketization, only in this way can sports dance develop better among the masses.

3) Sports dance teaching. Keyword clustering includes four clusters of labels 2, 3, 6 and 7, "Latin dance, training, competitive ability, investigation, University, teaching effect, teaching reform, action elements, rhythm teaching, school sports, sports dance teaching, teaching methods, teaching experience analysis, innovation and application, sports dance education, technical skill teaching, teaching content, music quality training, value Necessity, importance analysis, sports 
music, music knowledge ". In the four clusters of sports dance teaching, it mainly involves the training of Latin dance, the cultivation of sports dance music and the reform of sports dance teaching. Compared with the standard dance, Latin dance is more popular in China and is deeply loved by children, youth and college students. Based on this, some scholars have carried out research on the training of Latin dance. After consulting the relevant literature, it is found that the research on Latin dance training began in the 1990s. So far, there are also studies on Latin dance training. With the development of Latin dance in China, the competitive ability of Latin dance has become the top priority of Latin dance training. Through research, scholars collect the "strengths of a hundred schools", find out the training system suitable for Latin dance, and carry out targeted training for Latin dance, so as to improve the competitiveness of learners. In addition to the study of Latin dance training, there is also the study of sports dance teaching reform.

4) The cultural integration of national standard dance and Chinese classical dance. The keyword cluster is tag 4, in which the identification words mainly include "Chinese classical dance, national standard dance, musical, collaborative governance, cultural blending and localization". The dance introduced from abroad naturally has a unique foreign culture. When sports dance is introduced into China, it means that sports dance and Chinese local dance will inevitably go through cultural collision, then cultural combination, and finally cultural integration. Dance music is the best embodiment of their own culture. All kinds of dances have their own styles. While learning western music culture, only by integrating national music elements into sports dance can we blend sports dance with Chinese classical dance culture. In today's new era, we need to "localize" sports dance. We need to explore its value? Where is its dilemma? Where is the way out? We need follow-up researchers to conduct in-depth research, and finally we can really realize the "Sinicization" of sports dance.

5) The aesthetic value of sports dance. The key words are clustered into label 5, and the main identification words include "sports, art, aesthetics, teaching and training, characteristics, music beauty, expressive ability, body culture, body beauty, professional direction, professional players, educational concept, beauty, competitive value orientation, sports beauty, training objectives", etc. In the 1990s, some scholars discussed the appreciation and creation of human body beauty, clothing beauty, action beauty and music beauty in sports dance from the perspective of aesthetics. Because sports dance is a combination of sports and art, the aesthetic value of sports dance can not be ignored. Sports dance is a space-time art that expresses thoughts and feelings rhythmically through body movement. Through performance, the audience can have unlimited reverie and maximize the aesthetic value [5]. Through these papers, it is found that at the beginning, scholars mainly discussed the appreciation and aesthetic value of sports dance. Now, some scholars explore the aesthetic value of sports dance from the competitive aspect by exploring the sports competitive value of sports dance.

6) The influence of sports dance on College Students' physical and mental health. The keyword cluster is tag 8 , in which the identification words mainly include college students, entertainment, emotion, mental sub-health, fitness, dance therapy, interpersonal communication, coping style, healthy heart and beautiful body. Through the review of relevant literature, sports dance can promote college students' interpersonal communication ability and improve their mood [6]; Cultivate college students' team spirit [7]; It can promote the physical and mental health of college students and improve the mental sub-health state through intermediary effect [8]. Sports dance has its own unique fitness value, which can enhance students' physiological functions, fully adjust students' psychology and enter learning and life with a healthy attitude.

\section{Conclusion}

This study analyzes the hot topics in the field of sports dance research by consulting the relevant literature of sports dance and using CiteSpace software. The conclusions are as follows:

1) According to the number of documents issued, it can be divided into three time periods. The first stage is 1998-2009, and the overall number of documents issued is wavy and slowly upward; The second stage is 2010 , which is a sudden increase stage; The third stage is from 2011 to now. The annual number of documents decreased in waves, but the decline was small.

2) The cooperation between the authors is relatively scattered, but there are some small-scale cooperation of 2-3 people. Scholars who have great influence on sports dance research include leading figures represented by Huang Junya, Ma Dan, Zhang Qingshu and Chen Shude, which promote the development of sports dance in China. Through viewing the data, it is found that the representatives are mainly concentrated in Beijing Sports University.

3) The distribution of sports dance research institutions is scattered and the cluster scale is small, which leads to the weak sharing and mobility of sports dance related knowledge and research results. The research on sports dance mainly focuses on Sports Colleges and universities.

4) Through the keyword cluster analysis of sports dance research, the keywords are clustered into 9 cluster tags, each tag represents different meanings. Through sorting and summarizing, this study summarizes the nine clusters into six aspects: the development of sports dance, the development of sports dance in China's mass sports, sports dance teaching, the cultural integration of national standard dance and Chinese classical dance, the aesthetic value of sports dance, and the impact of sports dance on College Students' physical and mental health.

\section{References}

[1] Compilation Group of Sports Dance Course. Sports Dance Course[M]. Beijing: Beijing Sports University Press, 2016: 24.

[2] Sun Lin, Jin Yan. Development of Sports Dance Events in China[J]. Sports Culture Guide, 2013, 129 (3): 37-39. 
[3] Sun Lin, Ma Zhongli, Chen Guang. Comparative Study on the Development of Chinese and Foreign Sports Dance Brand Events[J]. Sports Culture Guide, 2013, 135(9): 37-40.

[4] Bu Jianhua. The Market Road of Sports Dance from the Perspective of Supply and Demand Theory[J]. Sports Culture Guide, 2013, 132(6): 32-34.

[5] Cui Xiaomei, Gao Hui. Exploring the beauty of Sports Dance. Journal of Northwest University (Philosophy and Social Sciences Edition), 2001(1): 156-159.

[6] Ma Gulandam, Liu Jian, Chen Zhailuzi, et al. Effect of Dance Therapy on Interpersonal Communication Ability and Emotion of College Students[J]. Journal of Shanghai Institute of Physical Education, 2019, 43(2): 86-90, 96.

[7] Cao Feifei, Wang Fei. Application of Sports Dance in Cultivating College Students' Team Spirit[J]. Journal of Physical Education, 2010, 17(6): 73-75.

[8] Yang Hui, Che Guangwei, Feng Yujuan, et al. The Impact of Sports Dance Exercise on College Students' Mental Sub-health: the Mediating Effect of Coping Styles[J]. Journal of Tianjin Institute of Physical Education, 2020, 35(5): 560-565.

\section{Author Profile}

Dacai Huang (1996 - ), Graduate student of Shenyang Sport University, Research direction: physical education. 